\title{
Erratum to: Pulsatile Perfusion Bioreactor System for Durability Testing and Compliance Estimation of Tissue Engineered Vascular Grafts
}

\author{
Stefanos E. Diamantouros, luis G. Hurtado-Aguilar, Thomas Schmitz-Rode, Petra Mela, \\ and Stefan JockenHOEvel \\ Department of Tissue Engineering \& Textile Implants, AME-Helmholtz Institute for Biomedical Engineering, Aachen \\ University, Pauwelsstrasse 20, 52074Aachen, Germany
}

Erratum to: Annals of Biomedical Engineering,

Vol. 41, No. 9, September 2013

(C) 2013) pp. 1979-1989

DOI 10.1007/s10439-013-0823-5

The supported grants were omitted from the Acknowledgment Section. The Acknowledgment section below is stated correctly:
Acknowledgments This research was carried out in the framework of the Transregio 37 project "Mikround Nanosysteme in der Medizin - Rekonstruktion biologischer Funktionen" funded by the German Research Foundation till 2011 and by the Else Kröner-Fresenius-Stiftung within the project 2011_A61.

Address correspondence to Stefan Jockenhoevel, Department of Tissue Engineering \& Textile Implants, AME-Helmholtz Institute for Biomedical Engineering, Aachen University, Pauwelsstrasse 20, 52074Aachen, Germany. Electronic mail: Jockenhoevel@hia. rwth-aachen. de

The online version of the original article can be found under doi: 10.1007/s10439-013-0823-5. 